\section{Estrategias para reducir la morbilidad y mortalidad de la diabetes ${ }^{1}$}

1 Basado en el documento "Strategies for reducing morbidity and mortality from diabetes through health-care system interventions and diabetes self-management education in community settings", elaborado por el Grupo de Estudio de Servicios Preventivos Comunitarios (Task Force on Community Preventive Services) y publicado en MMWR Morb Mortal Wkly Rep 2001; 50(RR16):1-15.
La diabetes es una enfermedad prevalente, con un alto costo y asociada a una considerable morbilidad y mortalidad. En 1997 se estimó que había en los Estados Unidos de América (EE. UU.) cerca de 16 millones de personas (aproximadamente un 6\% de la población) con diabetes y que la enfermedad no estaba diagnosticada en más de 5 millones de estas personas. La prevalencia de la diabetes aumenta con la edad (del 0,16\% en los menores de 20 años al $18,4 \%$ en los mayores de 65) y es más elevada en algunos grupos étnicos minoritarios. En 1996 la diabetes era la séptima causa de muerte en los EE. UU. En el grupo de 45 a 60 años, la mortalidad de los diabéticos duplica a la de los no diabéticos. La mortalidad relacionada con la diabetes se debe sobre todo a las cardiopatías, cuya tasa de mortalidad es 2 a 4 veces mayor en los diabéticos que en los no diabéticos. Lo mismo ocurre con el riesgo de accidentes cerebrovasculares. La diabetes es también la principal causa de ceguera en adultos de 20 a 74 años, y la principal causa de nefropatía terminal. Más del 50\% de las amputaciones realizadas en los EE. UU. tienen lugar en pacientes diabéticos. Las muertes relacionadas con el embarazo son dos veces más frecuentes en las mujeres diabéticas que en las no diabéticas. Los costos de la enfermedad son considerables y en 1997 se calculó que sus costos directos e indirectos en los EE. UU. ascendieron a 98 mil millones de dólares.

El control de la diabetes es complejo y difícil tanto para el paciente como para el profesional sanitario. Es difícil cambiar los estilos de vida (dieta, ejercicio, tabaco) y mantener los cambios a largo plazo. Las medicaciones, la inyección de insulina y la monitorización de la glucemia son complejas y molestas. El control de la diabetes requiere cantidades considerables de tiempo y dinero. Para alcanzar un control adecuado de la enfermedad, los diabéticos necesitan educación diabetológica y apoyo social.

La reducción de la morbilidad y mortalidad de la diabetes es un objetivo prioritario de la salud pública. Los objetivos fijados consisten en prevenir la diabetes, aumentar el diagnóstico temprano, aumentar las tasas de detección de las complicaciones y reducir la morbilidad y mortalidad. Para reducir la morbilidad y mortalidad, lo ideal es la prevención primaria. En pacientes con diabetes de tipo 2, la prevención consiste a menudo en el control del peso y la actividad física. 
Este informe proporciona orientaciones para todas las organizaciones y personas interesadas en mejorar la salud y el bienestar de los pacientes diabéticos, desde ellos mismos hasta las autoridades sanitarias estatales y locales o los responsables de la financiación de la salud pública.

\section{MÉTODOS}

El equipo que elaboró el documento se centró inicialmente en dos áreas prioritarias: las intervenciones en el sistema de salud para mejorar la asistencia a los diabéticos y las intervenciones educativas en el ámbito comunitario. Para ello se creó una lista de prioridades de intervenciones que deberían ser revisadas, teniendo en cuenta los siguientes criterios: 1) importancia de las intervenciones para reducir la morbilidad y mortalidad y mejorar la calidad de vida de los pacientes; 2) relación costoefectividad; 3) ausencia de datos claros sobre la eficacia de una intervención, y 4) factibilidad de la puesta en marcha de las intervenciones en la práctica diaria de la salud pública.

Para ser incluidos en las revisiones sobre la eficacia, los estudios tuvieron que cumplir los siguientes requisitos: 1) tratar de investigaciones primarias sobre las intervenciones seleccionadas; 2) haber sido publicados en inglés; 3) haber sido realizados en países con economías de mercado bien establecidas; 4) proporcionar información acerca de uno o más desenlaces de interés seleccionados previamente por el equipo, y 5) cumplir unos requisitos mínimos de calidad. Se incluyeron todos los tipos de estudios de diseño comparativo, como los ensayos clínicos aleatorizados controlados, las comparaciones antes y después de la intervención, las series temporales y los estudios de cohortes. Las evaluaciones realizadas en esta revisión se centraron en las pruebas sobre la eficacia de las intervenciones para mejorar las conductas de los pacientes y de los profesionales de la salud, y para mejorar los desenlaces de la enfermedad a corto y a largo plazo. Cada estudio que cumplió los criterios de inclusión fue examinado para determinar la calidad de su ejecución (buena, aceptable o escasa). Solo se incluyeron los de las dos primeras categorías. Las pruebas de la eficacia se clasificaron como firmes, suficientes o insuficientes, dependiendo del número de estudios existentes, de la idoneidad del diseño de los estudios para determinar la eficacia, de la calidad de su ejecución, de la coherencia de sus resultados y del tamaño del efecto. Las recomendaciones del Grupo de Estudio están basadas en las pruebas sobre la eficacia, esto es, se recomienda firmemente una intervención cuando las pruebas son firmes y se recomienda cuando las pruebas son suficientes. No obstante, la existencia de pruebas insuficientes sobre la eficacia de una intervención no debe ser interpretada como prueba de su ineficacia. En modificaciones futuras de estas recomendaciones también se tendrán en cuenta datos económicos.

\section{RESULTADOS}

Mediante búsquedas en bases de datos, revisión de las listas de referencias de los estudios incluidos y consultas con expertos en diabetes, se identificaron 105 estudios referidos a las siete intervenciones analizadas que cumplían los criterios de inclusión. Treinta y cinco de ellos fueron excluidos por problemas de calidad de ejecución. Las recomendaciones formuladas en este informe están basadas en los otros 70 estudios. De acuerdo con los resultados obtenidos, el Grupo de Estudio recomienda cuatro de las siete intervenciones investigadas, dos de ellas firmemente. Con respecto a las otras tres intervenciones, las pruebas obtenidas fueron insuficientes para poder hacer una recomendación a favor o en contra (cuadro 1).

\section{COMENTARIOS}

Al seleccionar y poner en práctica intervenciones como las analizadas, las comunidades y los sistemas de salud deberían desarrollar una estrategia global para promover estilos de vida sanos (más actividad física, mejor nutrición y reducción del consumo de tabaco, por ejemplo) y ayudar a los pacientes diabéticos y a los profesionales que los atienden a mejorar el control de la glucemia, a reducir las complicaciones y la mortalidad de la enfermedad y a mejorar la calidad de vida.

La elección de intervenciones de eficacia demostrada y bien ajustadas a las necesidades y capacidades locales y su puesta en marcha son pasos vitales para mejorar los desenlaces de los pacientes diabéticos. Si los objetivos y recursos locales lo permiten, se deberían iniciar o potenciar las intervenciones recomendadas. Antes que nada, las comunidades y los sistemas de salud deberían determinar la prevalencia de la diabetes y de sus complicaciones en su población y evaluar la calidad de la asistencia y el grado de educación diabetológica de los pacientes.

El Grupo de Estudio recomienda firmemente el control de la enfermedad y el control de casos para mejorar el sistema y los desenlaces de los pacientes. No obstante, hay que tener en cuenta que, para poner en práctica estas intervenciones, pueden 
CUADRO 1. Recomendaciones del Grupo de Estudio sobre las siete intervenciones analizadas

\begin{tabular}{|c|c|c|c|}
\hline $\begin{array}{c}\text { Intervención } \\
\text { (No. de estudios) }\end{array}$ & Descripción de la intervención & Resultados positivos & Recomendación \\
\hline $\begin{array}{l}\text { Control de la } \\
\text { enfermedad (25) }\end{array}$ & $\begin{array}{l}\text { Método de asistencia sanitaria organi- } \\
\text { zado, preventivo y con múltiples } \\
\text { componentes. La asistencia se cen- } \\
\text { tra en todo el espectro de la enfer- } \\
\text { medad y sus complicaciones, la pre- } \\
\text { vención de trastornos comórbidos y } \\
\text { los aspectos pertinentes del sistema } \\
\text { de atención } \\
\text { Duración mediana del seguimiento en } \\
\text { los estudios que investigaron la } \\
\text { HbG: } 18 \text { meses }\end{array}$ & $\begin{array}{l}\text { Mejoró la monitorización de la HbG } \\
\text { (15 estudios; mediana del efecto: } \\
\text { 15,6\%; IIC: } 4 \text { a } 39 \% \text { y los lípidos (9 } \\
\text { estudios; mediana del efecto: } 24 \% \text {; } \\
\text { IIC: } 21 \text { a } 26 \% \text { ) por el profesional de } \\
\text { la salud; las pruebas de detección } \\
\text { de la retinopatía ( } 15 \text { estudios; me- } \\
\text { diana del efecto: } 9 \% \text {; IIC: } 3 \text { a } 20 \% \text { ); } \\
\text { las lesiones de los pies y la neuro- } \\
\text { patía periférica (9 estudios; mediana } \\
\text { del efecto: } 26,5 \% \text {; IIC: } 10,9 \text { a } 54 \%) \text {, } \\
\text { y la proteinuria (7 estudios; mediana } \\
\text { del efecto: } 9,7 \% \text {; IIC: } 0 \text { a } 44 \%) \\
\text { Mejoró la HbG (19 estudios; mediana } \\
\text { del efecto: }-0,5 \% \text {; IIC: }-1,35 \text { a } \\
\text {-0,1\%) }\end{array}$ & $\begin{array}{l}\text { Firmemente } \\
\text { recomendada }\end{array}$ \\
\hline Control de casos (15) & $\begin{array}{l}\text { Conjunto de actividades destinadas a } \\
\text { identificar las necesidades de los } \\
\text { pacientes con riesgo de uso exce- } \\
\text { sivo de los recursos, desenlaces su- } \\
\text { bóptimos o coordinación subóptima } \\
\text { de los servicios y a suplir dichas ne- } \\
\text { cesidades mediante una mejor pla- } \\
\text { nificación, coordinación y prestación } \\
\text { de la asistencia. Puede funcionar } \\
\text { como intervención aislada, combi- } \\
\text { nada con otras intervenciones sobre } \\
\text { la asistencia clínica o como parte } \\
\text { de una intervención de control de } \\
\text { la enfermedad } \\
\text { Duración mediana del seguimiento en } \\
\text { los estudios que investigaron la } \\
\text { HbG: } 12,5 \text { meses }\end{array}$ & $\begin{array}{l}\text { Mejoró la frecuencia de la monitoriza- } \\
\text { ción de la HbG cuando se integró en } \\
\text { la intervención de control de la en- } \\
\text { fermedad ( } 5 \text { estudios; mediana del } \\
\text { efecto absoluto: } 33 \% \text {; IIC: }-7 \text { a } \\
60 \% \text { ). Mejoró las concentraciones } \\
\text { de HbG tanto cuando se integró en } \\
\text { la intervención de control de la en- } \\
\text { fermedad ( } 11 \text { estudios; cambio me- } \\
\text { diano de la HbG: }-0,5 \% \text {; IIC: }-0,65 \\
\text { a -0,46\%) como cuando se aplicó } \\
\text { aisladamente (3 estudios; cambio } \\
\text { mediano de la HbG: }-0,4 \% \text {; IIC: } \\
-0,6 \text { a -0,16\%) }\end{array}$ & $\begin{array}{l}\text { Firmemente } \\
\text { recomendada }\end{array}$ \\
\hline $\begin{array}{l}\text { Educación sobre el } \\
\text { autocontrol de la } \\
\text { diabetes en lugares } \\
\text { de reunión de la } \\
\text { comunidad (8) }\end{array}$ & $\begin{array}{l}\text { Educación diabetológica de mayores } \\
\text { de } 18 \text { años en lugares de reunión } \\
\text { de la comunidad (centros comunita- } \\
\text { rios, bibliotecas, instalaciones priva- } \\
\text { das no clínicas y centros religiosos) } \\
\text { Duración mediana del seguimiento } \\
\text { de la HbG: } 6 \text { meses }\end{array}$ & $\begin{array}{l}\text { El control de la glucemia mejoró en } 7 \\
\text { estudios. El tamaño del efecto com- } \\
\text { binado para la HbG fue de }-1,9 \% \\
\text { (intervalo de confianza del } 95 \% \text { : } \\
-2,4 \text { a }-1,4 \% \text { ) } \\
\text { La tensión arterial mejoró en } \\
2 \text { estudios }\end{array}$ & $\begin{array}{l}\text { Recomendada para } \\
\text { adultos con } \\
\text { diabetes de tipo } 2\end{array}$ \\
\hline $\begin{array}{l}\text { Educación sobre el } \\
\text { autocontrol de la } \\
\text { diabetes en el } \\
\text { domicilio (10) }\end{array}$ & $\begin{array}{l}\text { Educación diabetológica en el domici- } \\
\text { lio del paciente (visitas, instrucción } \\
\text { asistida por computadora, comuni- } \\
\text { cación electrónica con profesionales } \\
\text { de la salud) }\end{array}$ & $\begin{array}{l}\text { Diabetes de tipo 1: mejoró el control } \\
\text { de la glucemia en } 3 \text { de } 4 \text { estudios. } \\
\text { El tamaño del efecto combinado } \\
\text { para la } \mathrm{HbG} \text { fue de }-1,1 \% \\
\text { (intervalo de confianza del } 95 \% \text { : } \\
-1,6 \text { a }-0,6 \% \text { ) }\end{array}$ & $\begin{array}{l}\text { Recomendada para } \\
\text { niños y adolescen- } \\
\text { tes con diabetes } \\
\text { de tipo } 1\end{array}$ \\
\hline & $\begin{array}{l}\text { Duración mediana del seguimiento del } \\
\text { control de la glucemia en los diabéti- } \\
\text { cos de tipo } 1: 12 \text { meses } \\
\text { Duración mediana del seguimiento de } \\
\text { los diabéticos de tipo } 2: 12 \text { meses }\end{array}$ & $\begin{array}{l}\text { Diabetes de tipo 2: las pruebas obteni- } \\
\text { das fueron insuficientes para hacer } \\
\text { recomendaciones }\end{array}$ & $\begin{array}{l}\text { Pruebas insuficientes } \\
\text { para la diabetes } \\
\text { de tipo } 2\end{array}$ \\
\hline
\end{tabular}

Educación sobre e autocontrol de la de reunión de la comunidad (8) diabetes en el domicilio (10)
Conjunto de actividades destinadas a identificar las necesidades de los con riesgo de uso excebóptimos o coordinación subóptima de los servicios y a suplir dichas nesediante una mejor plalos estudios que investigaron la

Educación diabetológica de mayores de 18 años en lugares de reunión la comunidad (centros comunitaes privaDuración mediana del seguimiento ducación diabetológica en el domici(visitas, instrucción cación electrónica con profesionales

uración mediana del seguimiento de control de la glucemia en los diabétiuración mediana del seguimiento de los diabéticos de tipo 2: 12 meses studios; med IIC: 21 a $26 \%$ ) por el profesional de Mejoró la HbG (19 estudios; mediana del efecto: $-0,5 \%$; IIC: $-1,35$ a

Mejoró la frecuencia de la monitoriza de la $\mathrm{HbG}$ cuando se integró en la intervención de control de la enefecto absoluto: $33 \%$ : IIC: -7 a $60 \%)$. Mejoró las concentraciones de $\mathrm{HbG}$ tanto cuando se integró en mediano de la HbG: $-0,4 \%$; IIC:

control de la glucemia mejoró en 7 estudios. El tamaño del efecto comdo para la HbG fue de $-1,9 \%$ (intervalo de confianza del $95 \%$ :

a tensión arterial mejoró en

iabetes de tipo 1: mejoró el control en 3 de 4 estudios. para la $\mathrm{HbG}$ fue de $-1,1 \%$ (intervalo de confianza del $95 \%$ : $-0,6 \%$ das fueron insuficientes para hacer para la diabetes de tipo 2 
CUADRO 1. (Continuación)

\begin{tabular}{|c|c|c|c|}
\hline $\begin{array}{c}\text { Intervención } \\
\text { (No. de estudios) }\end{array}$ & Descripción de la intervención & Resultados positivos & Recomendación \\
\hline $\begin{array}{l}\text { Educación sobre el } \\
\text { autocontrol de la } \\
\text { diabetes en el lugar } \\
\text { de trabajo (1) }\end{array}$ & $\begin{array}{l}\text { Educación diabetológica en el lugar } \\
\text { de trabajo del paciente } \\
\text { Seguimiento: } 3 \text { meses }\end{array}$ & $\begin{array}{l}\text { Las pruebas obtenidas fueron insufi- } \\
\text { cientes para hacer recomendacio- } \\
\text { nes porque solo se identificó un } \\
\text { estudio }\end{array}$ & Pruebas insuficientes \\
\hline $\begin{array}{l}\text { Educación sobre el } \\
\text { autocontrol de la } \\
\text { diabetes en } \\
\text { campamentos } \\
\text { recreativos }(10)\end{array}$ & $\begin{array}{l}\text { Educación diabetológica en campa- } \\
\text { mentos recreativos } \\
\text { Duración mediana del seguimiento: } \\
\quad 4 \text { a } 6 \text { días }\end{array}$ & $\begin{array}{l}\text { Las pruebas obtenidas fueron insufi- } \\
\text { cientes para hacer recomendacio- } \\
\text { nes debido al número insuficiente de } \\
\text { estudios que evaluaron desenlaces } \\
\text { sanitarios pertinentes }\end{array}$ & Pruebas insuficientes \\
\hline $\begin{array}{l}\text { Educación sobre } \\
\text { el autocontrol de la } \\
\text { diabetes en la } \\
\text { escuela (1) }\end{array}$ & $\begin{array}{l}\text { Educación diabetológica del personal } \\
\text { de la escuela con el fin de mejorar } \\
\text { la salud y el bienestar de los niños } \\
\text { diabéticos } \\
\text { El programa educativo se centró en el } \\
\text { personal docente y en otro personal } \\
\text { de la escuela, pero se pudieron } \\
\text { medir variables de eficacia tanto en } \\
\text { el personal como en los alumnos } \\
\text { afectados } \\
\text { Seguimiento: } 6 \text { a } 8 \text { semanas }\end{array}$ & $\begin{array}{l}\text { Las pruebas obtenidas fueron insufi- } \\
\text { cientes para hacer recomendacio- } \\
\text { nes porque solo se identificó un } \\
\text { estudio }\end{array}$ & Pruebas insuficientes \\
\hline
\end{tabular}

a $\mathrm{HbG}$ : hemoglobina glucosilada.

b IIC: intervalo intercuartílico.

ser necesarios considerables recursos e infraestructura. Asimismo, se recomienda la educación sobre el autocontrol de la diabetes, en el domicilio para los niños y adolescentes, y en lugares de reunión de la comunidad para los adultos; las intervenciones en estos ámbitos no deben ser consideradas como sustitutas, sino como complemento de las realizadas en las clínicas, siendo fundamental una buena comunicación y colaboración entre los educadores y los profesionales de la salud.

\section{SYNOPSIS}

\section{Strategies to reduce diabetes morbidity and mortality}

This piece summarizes a document prepared by the Task Force on Community Preventive Services, an independent working group in the United States of America. The Task Force document presents an analysis of the effectiveness of seven interventions designed to improve diabetes control. Two of the seven are focused on health-care systems (disease and case management), and the five others are directed at persons with diabetes (diabetes self-management education delivered in the home, the workplace, community gathering places, recreational camps, and the school). After reviewing 70 studies on these seven interventions, there was sufficient evidence to recommend four of them: 1) disease management in health-care systems, 2) case management in health-care systems, 3) diabetes self-management education in community gathering places, for adults with type 2 diabetes, and 4) diabetes self-management education in the home, for children and adolescents with type 1 diabetes. 